\title{
Glucocorticoid-resistant asthma: pathogenesis and clinical implications for management
}

\author{
S.J. Szefler, D.Y.M. Leung
}

Glucocorticoid-resistant asthma: pathogenesis and clinical implications for management. S.J. Szefler, D.Y.M. Leung. @ERS Journals Ltd. 1997.

ABSTRACT: At the present time, emphasis is placed on viewing asthma as a manifestation of chronic airway inflammation, possibly secondary to allergen hypersensitivity. Consequently, one aspect of management is to institute measures of environmental control to minimize the inflammatory response related to allergen stimulation, and to administer anti-inflammatory therapy to resolve inflammation and prevent progression of disease.

Most patients respond very favourably to conventional therapy, as recommended in recent guidelines for asthma management. Some cases, however, remain very difficult to control despite high-dose inhaled glucocorticoids, even combined with oral glucocorticoid therapy. Management of these patients raises questions about the conditions that alter response to glucocorticoid therapy.

The patient with difficult to control asthma not only presents a challenge to clinical management but raises new questions concerning our ability to control the progression of disease. Is difficult to control asthma secondary to overwhelming or ongoing allergen exposure? Do anti-inflammatory medications, specifically inhaled glucocorticoids, really control the progression of the disease? Are these patients destined to become severe asthmatics at birth due to the inherent characteristics of their airways, or is this indeed a consequence of progressive inflammation?

This review will summarize present concepts of glucocorticoid-resistant asthma, current knowledge of the mechanisms of persistent inflammation, and the implications for management. The gaps in information will also be addressed in order to stimulate interest in further research that could lead to better understanding of the disease and potential windows for therapeutic intervention.

Eur Respir J 1997; 10: 1640-1647.
Divisions of Allergy \& Immunology and Clinical Pharmacology, Dept of Pediatrics, National Jewish Center for Immunology and Respiratory Medicine, and Dept of Pediatrics, University of Colorado Health Sciences Center, Denver, Colorado, USA.

Correspondence: S.J. Szefler

National Jewish Center for Immunology and Respiratory Medicine

1400 Jackson St

Dept of Pediatrics

Goodman Bldg., Rm. 926

Denver

Colorado 80206

USA

Keywords: Asthma, glucocorticoids

Received: December 161996 Accepted December 311996

Presented at the European Respiratory Society Meeting

Barcelona, Spain, September 20, 1995.

Supported by USPHS grants HL-36577, RR-00051, and AR-41256. S.J.S. holds the Helen Wohlberg \& Herman Lambert Chair in Pharmacokinetics.

\section{Clinical presentation of glucocorticoid-resistant asthma}

The condition of the majority of patients with asthma falls into the category of episodic, mild persistent, or moderate persistent as recently defined in the Global Initiative for Asthma [1]. Patients with severe persistent or "difficult to control" asthma are a small proportion of those individuals with asthma. However, they are clearly the most challenging for management, account for the majority of health care costs related to asthma management, and most importantly, are the patients who appear to be at high risk for asthma mortality. Asthma, therefore, appears to be a spectrum varying from a very mild, episodic, subclinical disease to one that is apparently "glucocorticoid (steroid) resistant (SR)", but remains responsive to bronchodilator therapy. Although not clearly documented, it appears that some patients in the difficult to control category go on to develop severe obstructive pulmonary disease, that is poorly reversible even with bronchodilator therapy.

Patients who are "glucocorticoid-resistant" have some or all of the following complicating features: exerciseinduced asthma; spontaneous severe, life-threatening exacerbations; frequent nocturnal exacerbations; and other concomitant medical disorders, such as sinusitis and gastro-oesophageal reflux. Although there is no single clinical definition of glucocorticoid-resistant asthma, patients are usually recognized by their difficulty in clinical management, frequent breakthrough symptoms, compromised quality of life, or near death episodes. Most of these patients also demonstrate significant adverse glucocorticoid effects secondary to high-dose and prolonged courses of treatment. Interestingly, some of these patients remain refractory to adverse glucocorticoid effects [2]. This observation raises questions regarding a localized versus a systemic refractoriness to glucocorticoids. Perhaps the former may be acquired, whilst the latter is a primary genetic defect in glucocorticoid response.

Certain conditions can interfere with the appropriate diagnosis and management of asthma. Conditions such as other respiratory disorders, environmental control, poor compliance, and inadequate therapy can be addressed by a careful medical evaluation and reorganization of the management plan (table 1). Patients who fail to respond prompt a more detailed evaluation and innovative approaches to treatment. These patients are referred 
Table 1. - Potential mechanisms for poor response to glucocorticoid therapy

Other respiratory disorder

Overwhelming allergen exposure

Irreversible airways hyperresponsiveness

Poor adherence to prescribed therapy

Inadequate dose of anti-inflammatory medication

Glucocorticoid pharmacokinetics

Rapid elimination

Poor distribution to site of action

Incomplete absorption of oral glucocorticoid

Immunological mechanisms contributing to persistent airway inflammation

Glucocorticoid desensitization

Abnormal glucocorticoid receptor or postreceptor phenomenon

to as "difficult to control" if they require high-dose inhaled and oral glucocorticoid therapy, $>20 \mathrm{mg}$ every second day or $10 \mathrm{mg} \cdot \mathrm{day}^{-1}$ oral prednisone, or "glucocorticoid-resistant" if they appear refractory to higher doses of oral glucocorticoid therapy.

Our operational definition for this group of patients, termed "glucocorticoid-resistant," includes those patients who have a prebronchodilator morning forced expiratory volume in one second $(\mathrm{FEV} 1)<70 \%$ of predicted, and who fail to increase this pre-bronchodilator morning FEV1 measurement by $15 \%$ after at least 1 week of an oral glucocorticoid course consisting of prednisone $40 \mathrm{mg} \cdot$ day $^{-1}$. They must also demonstrate a $15 \%$ improvement in FEV1 following a rapidly-acting bronchodilator treatment. Other investigators differ in their operational definition for glucocorticoid-resistant asthma. For example, some will begin with a course of prednisolone, 20 $\mathrm{mg} \cdot \mathrm{day}^{-1}$ for 1 week, and then increase it to $40 \mathrm{mg} \cdot \mathrm{day}^{-1}$ for a second week, before declaring the patient glucocorticoid-resistant [3]. Patients with "difficult to control" asthma are not necessarily "glucocorticoid-resistant." A proportion of patients who are identified as glucocorticoid-resistant will improve their clinical response if aggressive therapy is extended beyond 2 weeks, but this course of treatment places the patient at risk for significant adverse effects commonly associated with glucocorticoid therapy.

\section{Persistent immune activation as a component of glucocorticoid-resistant asthma}

SCHWARTZ et al. [4] first reported the observation of apparent glucocorticoid resistance in asthma management. They recognized a reduced eosinopenic response and accelerated plasma cortisol clearance in glucocorticoidresistant asthmatic patients as compared to a control population. The initial observation of a reduced eosinopenic response to glucocorticoids has not been evaluated in an expanded population of glucocorticoid-resistant patients since they are routinely managed with high-dose oral glucocorticoid therapy. Patients receiving high-dose oral glucocorticoids usually have suppressed morning plasma cortisol concentrations. Some of these patients, however, retain low or, more rarely, normal morning plasma cortisol concentrations despite daily oral glucocorticoid therapy [2]. Compliance must certainly be verified in this group of patients before embarking on a course of intricate laboratory investigation.

\section{Glucocorticoid pharmacokinetics}

If a patient fails to respond or is unable to tolerate glucocorticoid doses lower than $20 \mathrm{mg}$ every second day with either prednisone or methylprednisolone, we usually evaluate glucocorticoid pharmacokinetics. The purpose of this evaluation is to determine whether there is incomplete glucocorticoid absorption, failure to convert an inactive form (prednisone) to an active form (prednisolone), or rapid elimination. The evaluation is particularly important in a patient who fails to demonstrate the anticipated adverse effects [5]. Measurements of plasma glucocorticoid concentrations can also be used in an assessment of compliance. Although the majority of patients have normal absorption, conversion to the active form, and elimination, a proportion of these patients have pharmacokinetic abnormalities [6,7]. The most frequent finding is rapid elimination, usually secondary to a drug-drug interaction, particularly the concomitant use of anticonvulsants, such as phenytoin sodium, carbamazepine or phenobarbitone. Occasional patients may show poor absorption and, interestingly, substitution with an alternative glucocorticoid will show improved response [5].

\section{Analysis of peripheral blood mononuclear cells}

Our own studies have confirmed that glucocorticoid resistant asthmatics have a glucocorticoid dose response curve shifted to the right using a functional measure of glucocorticoid-response, specifically phytohaemagglutinin-induced inhibition of peripheral blood mononuclear cell (PBMC) proliferation [8]. Other investigators have identified a similar alteration in the dose-response curve to a topical glucocorticoid with a skin blanching technique [9]. This prompted investigation of glucocorticoid receptor-binding in these patients.

The importance of the glucocorticoid receptor (GCR) in determining clinical responsiveness to glucocorticoids was reported previously as primary cortisol resistance in association with an endocrine abnormality [10]. These patients presented with elevated total plasma cortisol concentrations, but no cushingoid features. The molecular basis for glucocorticoid resistance was eventually attributed to reduced GCR numbers, decreased ligandbinding affinity for glucocorticoids, or overexpression of a mutated GCR [11]. The latter phenomenon results in a GCR which binds the ligand with a much lower affinity than the wild type, leading to reduced expression of a glucocorticoid-responsive reporter gene (MMTVLTR CAT). As such, we felt it was important to determine whether the poor glucocorticoid responses in SR asthma resulted from an alteration in GCR number or binding affinity.

In this regard, we have identified several GCR abnormalities associated with SR asthma [2]. In one study, a $\left[{ }^{3} \mathrm{H}\right]$-dexamethasone radioligand-binding assay and Scatchard analysis was used to evaluate PBMCs from 17 patients with SR asthma: 15 of the asthmatics had a significant increase in their GCR dissociation constant (Kd), i.e. a decrease in binding affinity for glucocorticoids as compared to normal subjects or SR asthmatics. This defect, attributed to reduced GCR binding affinity, was designated as Type I SR asthma. Two other patients 
with SR asthma had normal GCR Kd values but a significantly decreased number of GCR binding sites per cell. The latter defect was designated as Type II SR asthma.

Next, it was of interest to determine whether the altered GCR binding parameters were restricted to specific subsets of PBMCs. Therefore, GCR binding parameters in their T-cell versus non-T-cell subpopulations were evaluated. A fourfold increase in T-cell, as compared to non-T-cell, GCR Kd was observed in PBMCs from Type I SR asthmatics [2]. The T-cells from Type I SR asthmatics also had a significantly higher GCR Kd than T-cells from normal controls. Alternatively, the abnormally low GCR number observed in Type II SR asthma was present both in T-cells and non-T-cells.

SR asthmatics frequently have a history of long-term glucocorticoid therapy. Thus, concern has been raised that the abnormal GCR binding observed in SR asthma may be a result of systemic glucocorticoid therapy. Several observations suggest that this is not the case. Firstly, a number of patients presenting with Type I (decreased GCR binding affinity) SR asthma have not previously received oral prednisone [2]. Secondly, in a group of SR asthmatics not receiving oral prednisone therapy, no significant change in GCR binding parameters was observed following a 1 week course of high-dose prednisone [2]. Thirdly, the same level of abnormal GCR binding affinity as found in SR asthmatics was not observed in patients with interstitial lung disease, receiving chronic high-dose prednisone therapy [2]. Fourth, at least one study reported that glucocorticoid treatment induces a modest downregulation of GCR number, which is different from the observed GCR abnormality in Type I SR asthma [12]. Finally, we examined GCR binding affinity in 10 patients with poorly controlled asthma prior to and after a course of high-dose prednisone therapy. These patients demonstrated decreased GCR binding affinity at baseline. Subsequently, after prednisone treatment, the presumed reduction in airway inflammation was associated with a significant decrease in GCR Kd toward normal values [13].

Evidence, so far, suggests there is only one human GCR gene, which exists as a single copy [14]. We have observed that patients with Type I SR asthma often have severe side-effects from chronic treatment with systemic glucocorticoids, and that their GCR defect is restricted to T-cells. These observations suggest that Type I SR asthma is acquired. Since Type II SR asthma does not appear to be associated with the development of glucocorticoid-induced side-effects and is not limited to Tcells, we feel this defect may be analogous to patients with primary cortisol resistance and would, therefore, be expected to be an irreversible GCR defect. The latter group of patients requires further investigation in order to resolve the mechanism of their abnormality.

To determine whether the reduced GCR binding affinity in PBMCs from Type I SR asthmatics was reversible, GCR binding was measured in PBMCs prior to and following incubation for $48 \mathrm{~h}$ in culture medium. When PBMCs from these patients were incubated in medium they showed a significant increase in GCR binding, with normalization of GCR Kd after $48 \mathrm{~h}$ of incubation [2]. Incubation of PBMCs from normal donors under similar culture conditions, however, did not result in any significant change in GCR-binding. We, therefore, concluded that the decreased GCR-binding affinity found in T-cells from patients with Type I SR asthma is an acquired defect. This is in agreement with the observations of other investigators [15], that there is no polymorphism within the functional domains of GCR complementary deoxyribonucleic acid (cDNA) in patients with SR asthma.

It is our impression that abnormalities in GCR binding do not appear to be the sole reason for difficult to control asthma, but these abnormalities in binding do seem to indicate that response to glucocorticoid therapy is affected by inflammation, and these patients appear to have persistent inflammation. Information is needed on mechanisms for persistent inflammation and the actual concentrations of glucocorticoid present at relevant sites of inflammation.

\section{Molecular mechanisms for glucocorticoid resistance}

The mechanisms by which cytokines, specifically the combination of interleukin (IL)-2 and IL-4, result in decreased glucocorticoid responsiveness in asthma are not defined. One possible insight is that the GCR ligandbinding abnormality in Type I SR asthmatics is localized to the nuclear GCR, but not cytosolic GCR [2, 16]. The GCR is known to change its structure and conformation when translocated between the cytosol and nucleus [17]. The activated GCR nuclear complex regulates transcription when it binds to specific DNA glucocorticoid response elements (GREs). The induction or repression of the GCR target genes then results in the altered expression of glucocorticoid regulated proteins [18]. This step is mediated by direct binding of the modulatory domain of the GCR with transcription factors, for example activating protein-1 (AP-1) or nuclear factor- $\kappa \mathrm{B}(\mathrm{NF}-\kappa \mathrm{B})$. Overexpression of AP-1 or NF- $\mathrm{B}$, however, in-terferes with GCR function as a result of direct protein-protein interactions between these transcription factors and the GCR $[19,20]$. Since SR asthma is associated with higher levels of cytokine activation than those observed in patients with glucocorticoid (steroid) sensitive (SS) asthma, the activation of specific transcription factors, such as AP-1 or NF- $\kappa \mathrm{B}$, may be an explanation for the nuclear localization of the GCR-binding defect in SR asthma and differences in patterns of glucocorticoid responsiveness in asthma.

These observations have suggested the possibility that, in addition to decreased GCR ligand-binding, PBMCs from patients with SR asthma may also have decreased binding of their GCR to DNA GREs. Recently, ADCOCK et al. [20] employed electrophoretic mobility shift assays to evaluate binding of nuclear translocated GCR from PBMCs of SR and SS asthmatics to GRE DNA binding sites. Dexamethasone induced a twofold increase in GRE binding in PBMCs from SS asthmatics and nonatopic controls, but this was markedly reduced in SR asthmatics. Analysis of GCR-GRE binding showed no change in binding affinity but did show a reduced number of GCRs available for DNA binding. These results suggest that GCR binding to GRE was impaired in monocytes from SR asthmatics. We have also found that peripheral 
blood lymphocytes from patients with SR asthma can have GRE DNA binding abnormalities [21]. Thus, there may be considerable heterogeneity in the GCR defects which result in impaired glucocorticoid responsiveness in SR asthma. Another explanation that deserves further investigation is the potential induction of GCR $\beta$, a result of alternative splicing of the GCR pre-messenger ribonucleic acid (mRNA), which acts as an endogenous inhibitor of GCR DNA binding [22].

Since our experience has indicated that the majority of patients with SR asthma have an acquired form of glucocorticoid resistance induced by inflammation or immune activation, we were interested in determining whether factors, such as allergen exposure, known to contribute to poorly controlled asthma, would have an effect on GCR binding affinity. In support of this concept of glucocorticoid resistance induced by inflammation, LANE et al. [23] recently reported that patients with glucocorticoid-resistant asthma do not have an altered secretory rate of endogenous cortisol or an altered sensitivity of the hypothalamic-pituitary-adrenal axis to dexamethasone suppression. As a result, we recently examined whether exposure to allergens alter GCR binding affinity in PBMCs from atopic asthmatics [24]. PBMC GCR Kd values from 12 ragweed-sensitive asthmatics were measured prior to, during the peak of, and after the ragweed season. A significant reduction in the GCR binding affinity was noted during the ragweed pollen season, as compared to that obtained prior to and after the ragweed season.

In vitro effects of allergen treatment on GCR $\mathrm{Kd}$ were also examined with PBMCs from atopic asthmatics by incubating their cells for $48 \mathrm{~h}$ using ragweed or cat allergen. GCR binding affinity was significantly reduced after a 48 hour incubation with ragweed or cat allergen, as compared to the respective baseline measurement.

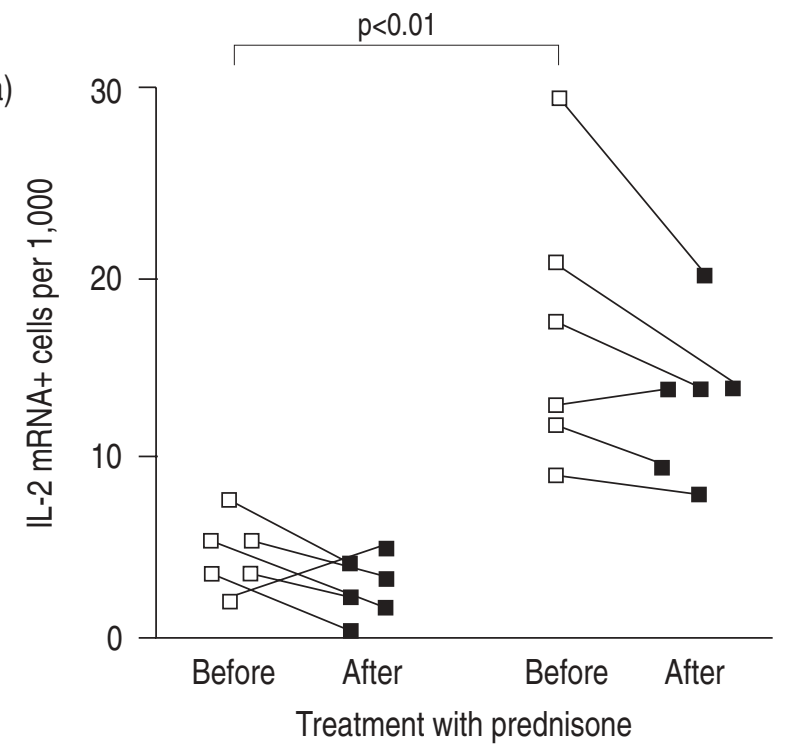

These effects were considered to be allergen-specific because Candida albicans had no such effect on GCR $\mathrm{Kd}$. These observed allergen-induced reductions in GCR binding affinity also resulted in significantly reduced inhibitory effects to dexamethasone and hydrocortisone on T-cells from atopic asthmatics. Overall, these observations suggest that allergen exposure is likely to contribute to poor asthma control by reducing GCR binding affinity in T-lymphocytes. Further studies are needed to identify other mechanisms by which allergens result in altered glucocorticoid responses, as well as other triggers, such as infectious agents, which may contribute to this process.

\section{Airways inflammation}

This area of investigation in glucocorticoid-resistant asthma has undergone limited evaluation. It is particularly important to conduct investigations on the effects of glucocorticoids on the airway cells in patients with SR asthma. In this regard, we recently examined cytokine gene expression of bronchoalveolar lavage (BAL) cells from SS and SR asthmatics, before and after a 1 week course of oral prednisone, $40 \mathrm{mg} \cdot \mathrm{day}^{-1}$ [25]. Prior to prednisone therapy, BAL cells from patients with SR, as compared to SS, asthma had significantly higher numbers of cells expressing mRNA for IL-2 and IL-4 (fig. 1). There was no significant difference between these two patient populations in their expression of IL-5 mRNA and interferon- $\gamma($ IFN- $\gamma)$ mRNA prior to prednisone therapy. Following the course of prednisone therapy, BAL cells from SS asthmatics demonstrated a significant decrease in the number of cells expressing mRNA for IL4 and IL-5. Prednisone therapy in patients with SR asthma did not alter the expression of IL-4 or IL-5

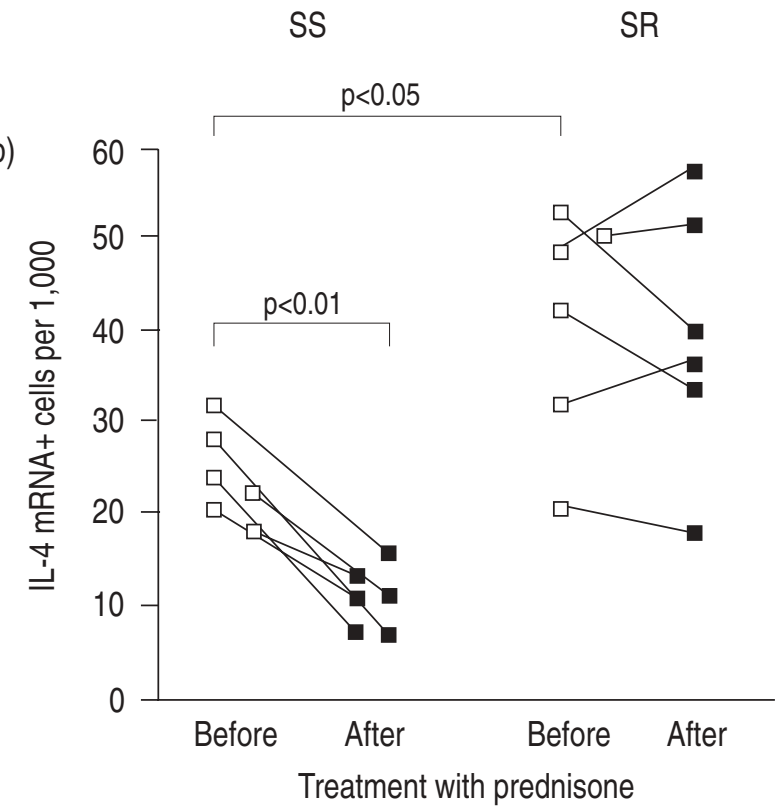

Fig. 1. - Number of cells with positive hybridization signals for: a) interleukin-2 (IL-2) mRNA; and b) interleukin-4 (IL-4) mRNA in bronchoalveolar (BAL) fluid from six glucocorticoid (steroid) sensitive (SS) asthmatics and six glucocorticoid (steroid) resistant (SR) asthmatics obtained prior to and after treatment for 1 week with prednisone $40 \mathrm{mg} \cdot \mathrm{day}^{-1}$. Prior to prednisone therapy, there were significantly more cells expressing IL-2 $(\mathrm{p}<0.01)$ and IL-4 $(\mathrm{p}<0.05)$ mRNA in the BAL fluid of patients with SR asthma as compared to SS asthma. mRNA: messenger ribonucleic acid. (Reproduced with permission from [25]). 
mRNA in their airways. In addition, small reductions in the numbers of BAL cells per 1,000 positive for IL-2 mRNA accompanied prednisone therapy in each patient group, however, these differences were not significant.

Prednisone treatment of SS asthmatics resulted in a significant increase in the number of BAL cells expressing IFN- $\gamma$ mRNA [11]. When SR asthmatics were treated with prednisone, however, their BAL cells demonstrated a decrease in IFN- $\gamma$ mRNA+ cells. These divergent responses in IFN- $\gamma$ gene expression following prednisone treatment resulted in a highly significant difference between the SR versus SS asthmatic groups. Therefore, the airway cells from the patients with SR asthma, as compared to those with SS asthma, show different patterns of cytokine gene expression, and show distinct responses to glucocorticoid therapy on cytokine gene expression in specific cells or on overall cell migration. It is possible that these varying cell patterns of cytokine gene expression contribute to the pathogenesis of asthma.

\section{Approach to management}

Some patients with apparent glucocorticoid-resistant asthma are inadequately managed due to their own noncompliance with recommended treatment or inadequate attention to environmental control measures and aggressive anti-inflammatory therapy. The general principles of management for these patients are summarized in table 2, and include the following:

1. Obtain careful history regarding relevant features of severe asthma and concomitant medical disorders, e.g. sinusitis and gastro-oesophageal reflux.

2. Assure appropriate environmental control, including school and work. Focus on areas where the patient spends the greatest time, e.g. the bedroom, or areas of high allergen exposure.

3. Incorporate objective measures, i.e. spirometry on

Table 2. - Approach to the management of glucocorticoid-resistant asthma

Examine for concomitant medical disorders, e.g. sinusitis and gastro-oesophageal reflux

Environmental control

Monitor pulmonary function

Written action plan

Review medication technique

Control nocturnal exacerbations

Salmeterol

Theophylline

High-dose inhaled glucocorticoid therapy

New inhaled glucocorticoids

Budesonide

Fluticasone

Oral glucocorticoid therapy

Evaluate glucocorticoid pharmacokinetics and receptors

Methylprednisolone

Split-dosing regimen

$15.00 \mathrm{~h}$ dosing

Monitor for adverse effects

Cyclosporin

Methotrexate

Gold

Intravenous gammaglobulin office visits and routine peak flow monitoring. Peak flow measurements can be particularly useful in identifying diurnal variations in pulmonary function and assessing the response to therapeutic intervention and adjustments of medication. For children, a lung growth chart may be useful in following the course of treatment.

4. Develop a written action plan for acute exacerbations. Emphasis should be placed on appropriate use of bronchodilators and when to notify the physician. A written care plan should also be developed to summarize routine medications, including recommendations for pretreatment programmes for exercise, and anticipated exposure to irritants or allergens. If a patient has difficulty in following the recommendations or appears to be intentionally noncompliant, a psychological evaluation could be helpful in identifying psychosocial features that interfere with adherence to the treatment regimen, for example, family dysfunction, learning disorders, depression, anxiety, etc.

5. Monitor the technique of administration of medication, incorporating this as a routine part of the physical examination. Utilize spacer devices to optimize delivery of medication. For inhaled glucocorticoids, advise mouth rinsing and expectoration of mouth rinse to minimize systemic glucocorticoid absorption. Explore the use of various delivery devices as they become available, e.g. dry powder breath-actuated systems.

6. Maximize anti-inflammatory therapy and reserve bronchodilator therapy for rescue treatment and nocturnal exacerbations. Salmeterol and theophylline can be very useful in controlling nocturnal asthma. Apply chronopharmacological principles to optimize response to theophylline, for example patients with nocturnal exacerbations may do much better with a single dose of a once daily sustained release preparation administered in the evening, as compared to a standard twice daily preparation [26, 27]. Children and rapid theophylline metabolizers appear to be prone to reduction in serum theophylline concentrations during the night [28]. This is likely to be related to the imbalance of rapid elimination and slow absorption (secondary to posture effects on absorption). We occasionally incorporate evaluations of $24 \mathrm{~h}$ monitoring to assist in developing an individualized treatment schedule [28].

7. To maximize pulmonary function with oral glucocorticoids, consider a split-dosing regimen, with the second dose administered in the afternoon. Titrate the morning dose, then convert the afternoon dose to the morning dose, and then attempt to reduce to alternate day therapy. Whilst several single dose studies have indicated that maximum response to systemic glucocorticoid therapy occurs when the dose is administered in the afternoon, there are no multiple dose, long-term studies to verify efficacy and safety as compared to standard treatment protocols [29].

If a patient fails to respond or is unable to tolerate glucocorticoid doses lower than $20 \mathrm{mg}$ every other day with either prednisone or methylprednisolone, we evaluate glucocorticoid pharmacokinetics and glucocorticoid receptors. The purpose of these studies is to determine whether there is incomplete glucocorticoid absorption, failure to convert to an active form, rapid elimination, reduced GCR number or binding affinity, or a combination of abnormalities. This evaluation is particularly important 
in a patient who fails to demonstrate the anticipated adverse effects of long-term, high-dose glucocorticoid therapy $[2,5]$. Measurements of plasma glucocorticoid concentrations can also be used in an assessment of compliance. We are also beginning to incorporate markers of inflammation, for example, plasma eosinophilic cationic protein (ECP) and serum soluble interleukin-2 receptors (sIL-2), to examine response to medication. This is most useful before and after a 1-2 week course of oral glucocorticoid therapy. Failure to respond provides a strong base for incorporating alternative therapies. We are beginning to obtain experience with measurements of exhaled nitric oxide as a measure of inflammation and response to therapeutic intervention [30].

8. Patients must be monitored carefully for adverse effects related to glucocorticoid therapy and measures taken to minimize such effects. For example, glucocorticoid-induced osteoporosis can be monitored with bone densitometry. Attention should be given to providing adequate dietary calcium and vitamin D, as well as other therapeutic interventions, as indicated. Based on the level of severity, we incorporate specialists in bone metabolism to assist in managing this potentially devastating adverse effect.

Alternative treatment options are a source of concern for patients with glucocorticoid-resistant asthma. Limited information from in vitro studies suggests that cyclosporin could be a useful agent in the management of these patients [31]. At the present time, no specific study focusing on patients with glucocorticoid-resistant asthma is available. Before attempting treatment with cyclosporin, several therapeutic options should be considered.

An obvious approach to the management of severe asthma is to increase the dose and frequency of inhaled glucocorticoids, presuming that higher doses would be more effective and also that adverse effects would be less than those commonly associated with high-dose systemic glucocorticoid therapy. Unfortunately, there is very little information on the safety and efficacy of inhaled glucocorticoids at doses exceeding those recommended in the product literature. A dose-response study conducted with budesonide provides some insight into this question. The results of this study indicate that, in children with moderate-to-severe asthma, doses less than $400 \mu \mathrm{g} \cdot \mathrm{day}^{-1}$ are sufficient to maximize pulmonary function, however, higher doses given over extended periods of time may be needed to alleviate other symptoms, such as exercise-induced asthma [32]. Information is needed on the comparative efficacy and safety of the various inhaled glucocorticoids with relevant clinical parameters, and the long-term safety of inhaled glucocorticoids, especially at higher than recommended doses.

Two new inhaled glucocorticoids, budesonide and fluticasone, are likely to be approved for use in the United States within the next year. Both are characterized by high topical potency, rapid clearance once absorbed, low oral bioavailability, high affinity to the glucocorticoid receptor, and relatively inactive metabolites. The most detailed information on inhaled glucocorticoid pharmacokinetics is available for budesonide and fluticasone [33]. At the present time, budesonide may have an additional benefit with the availability of a dry powder, breath-actuated delivery device (Turbuhaler; Astra, Lund,
Sweden). This improves pulmonary deposition and, consequently, may be responsible for the oral glucocorticoid-sparing effect observed with this medication. Fluticasone also has very high affinity to the glucocorticoid receptor, and this may explain its benefit in contributing to a significant oral glucocorticoid reduction in patients with severe asthma [34]. Because of the high GCR binding with fluticasone propionate, further studies are needed on risks for systemic adverse effects, especially at high doses, e.g. doses exceeding 2,000 $\mu \mathrm{g} \cdot \mathrm{day}^{-1}$.

It is important to compare all available inhaled glucocorticoids and all new forms under a standard set of conditions, in order to fully quantitate relative systemic availability and risk for systemic effects. One study suggests that the three inhaled glucocorticoids available in the United States, beclomethasone dipropionate, triamcinolone acetonide and flunisolide, are equal in efficacy and systemic adverse effects when compared on a dose per dose basis [35]. Therefore, this study concludes that the relative potency per inhalation is approximately proportional to the dose delivered.

For now, it appears that all currently available inhaled glucocorticoids in the United States are similarly effective at low doses [36]. Whether or not they differ in safety and efficacy at higher doses in more severe asthmatics is unknown. The most detailed studies in patients with severe asthma have been reported for budesonide and fluticasone. Both are effective in reducing oral prednisone requirements [34, 37]. Further studies must compare the relative efficacy and toxicity in patients with glucocorticoid resistant asthma.

Equally important is the effect of new delivery devices on pulmonary deposition as well as systemic absorption of inhaled glucocorticoids. The studies available suggest that pulmonary deposition is increased approximately twofold with the new dry powder breath-actuated delivery devices, such as the Turbuhaler. While this increases clinical efficacy, it also increases the amount of drug absorbed per actuation $[38,39]$. These studies indicate that different dosing strategies, based on the type of glucocorticoid and the type of delivery device, will be necessary to optimize clinical efficacy and minimize the risk of adverse effects.

Alternative anti-inflammatory and immunomodulator approaches, such as methotrexate, gold, cyclosporin, and intravenous gammaglobulin, have been poorly studied in difficult to control asthma and there are no studies in patients with glucocorticoid resistant asthma. The studies available have failed to demonstrate resolution of inflammation [40, 41]. Most of these medications have been identified by chance or extrapolated for use in asthma based on efficacy in other inflammation-based diseases, such as rheumatoid arthritis. Of interest is the fact that none of these medications have incorporated a study with bronchial biopsy and BAL to verify resolution of inflammation. In studies where an indirect measure of inflammation, i.e. airways hyperresponsiveness, was measured, no change was observed (methotrexate, intravenous gammaglobulin). An organized programme with carefully designed protocols and larger numbers of patients is obviously needed to pursue these preliminary observations and to identify a hierarchy for selection of medication in patients with severe asthma. 
Several new classes of medication are presently undergoing clinical evaluation and could be available within the next 2 yrs. These include the 5-lipoxygenase inhibitors and leukotriene (LT) antagonists. Peptidoleukotrienes are associated with a number of features relevant to the pathogenesis of asthma, including increased mucus secretion, increased vascular permeability, and smooth muscle contraction. The enzyme pathway for leukotriene synthesis can be effectively inhibited by zilueton, a 5-lipoxygenase inhibitor, that reduces the synthesis of $\mathrm{LTC}_{4}, \mathrm{LTD}_{4}, \mathrm{LTE}_{4}$ and $\mathrm{LTB}_{4}$. In addition, $\mathrm{LTD}_{4}$ activity can be inhibited by another compound, accolate (ICI 204,219). The latter will also have an effect in blocking $\mathrm{LTC}_{4}$ and $\mathrm{LTE}_{4}$, but not $\mathrm{LTB}_{4}$. The clinical studies available show effects of both drugs on blocking bronchospasm due to cold air, exercise and aspirin, as well as allergen challenge [42-44]. Regular administration can also improve pulmonary function and asthma control.

More information is needed on the pathology of difficult to control asthma to determine whether there are ultrastructural abnormalities present that may be irreversible [45]. It is possible that aggressive courses of anti-inflammatory or immunomodulator therapy can suppress active inflammation, but airway remodelling may predispose the patient to residual symptoms secondary to persistent airways hyperresponsiveness. Of greater concern is the possibility that the persistent symptoms in certain patients could be related to noninflammatory airways hyperresponsiveness. Obviously, more effort must be placed on understanding the pathophysiology of severe asthma to refine the selection of pharmacotherapy for this challenging group of patients.

Acknowledgement: The authors wish to thank M. PlourdSandoval for assistance in preparing this manuscript.

\section{References}

1. Global Initiative for Asthma. Global strategy for asthma management and prevention, NHLBI/NIH workshop report. National Institutes of Health, National Heart, Lung, and Blood Institute Publication No. 95-3659, 1995.

2. Sher ER, Leung DYM, Surs W, et al. Steroid resistant asthma: cellular mechanisms contributing to inadequate response to glucocorticoid therapy. J Clin Invest 1994; 3: 33-39.

3. Barnes PJ, Greening AP, Crompton GK. Glucocorticoid resistance in asthma. Am J Respir Crit Care Med 1995; 152: S125-S142.

4. Schwartz HJ, Lowell FC, Malby JC. Steroid resistance in bronchial asthma. Ann Intern Med 1968; 69: 493499.

5. Hill MR, Szefler SJ, Ball BD, Bartoszek M, Brenner M. Monitoring glucocorticoid therapy: A pharmacokinetic approach. Clin Pharmacol Ther 1990; 48: 390398.

6. Corrigan CJ, Brown P, Barnes NC, et al. Glucocorticoid resistance in chronic asthma: glucocorticoid pharmacokinetics, glucocorticoid receptor characteristics and inhibition of peripheral blood T-cell proliferation by glucocorticoid in vitro. Am Rev Respir Dis 1991; 144: 1016-1025.

7. Kamada AK, Spahn JD, Surs W, Brown E, Leung DYM, Szefler SJ. Coexistence of glucocorticoid receptor and pharmacokinetic abnormalities: factors that contribute to a poor response to treatment with glucocorticoids in children with asthma. J Pediatr 1994; 124: 984-986.

8. Alvarez J, Surs W, Leung DYM, Iklé D, Gelfand EW, Szefler SJ. Steroid-resistant asthma: immunologic and pharmacologic features. J Allergy Clin Immunol 1992; 89: 714-721.

9. Brown PH, Teelucksingh S, Matusiewicz SP, Greening AP, Crompton GK, Edwards CRW. Cutaneous vasoconstrictor response to glucocorticoids in asthma. Lancet 1991; 337: 576-580.

10. Chrousos GP, Vingerhoeds A, Brandon D, et al. Primary cortisol resistance in man: a glucocorticoid receptormediated disease. J Clin Invest 1982; 69: 1261-1269.

11. Hurley DM, Accili D, Stratakis CA, et al. Point mutation causing a single amino acid substitution in the hormone-binding domain of the glucocorticoid receptor in familial glucocorticoid resistance. J Clin Invest 1991; 87: 680-686.

12. Vedeckis WV, Ali M, Allen HR. Regulation of glucocorticoid receptor protein and mRNA levels. Cancer Res 1989; 49: 2295s-2302s.

13. Spahn JD, Leung DYM, Surs W, Nimmagadda SR, Szefler SJ. Effect of prednisone treatment on glucocorticoid receptor-binding parameters in poorly controlled asthma. Am J Pediatr Asthma Allergy 1994; 7: 87-92.

14. Hollenberg SM, Weinberger C, Ong ES, et al. Primary structure and expression of a functional human glucocorticoid receptor cDNA. Nature 1985; 318: 635-641.

15. Lane SJ, Arm JP, Staynov DZ, Lee TH. Chemical mutational analysis of the human glucocorticoid receptor cDNA in glucocorticoid-resistant bronchial asthma. Am J Respir Cell Mol Biol 1994; 11: 42-48.

16. Kam J, Szefler SJ, Surs W, Sher E, Leung DYM. The combined effects of IL-2 and IL-4 alter the binding affinity of the glucocorticoid receptor. I Immunol 1993; 151: 3460-3466.

17. Munck A, Mendel DB, Smith LI, Orti E. Glucocorticoid receptors and actions. Am Rev Respir Dis 1990; 141: S2-S10.

18. Truss M, Beato M. Steroid hormone receptors: interaction with deoxyribonucleic acid and transcription factors. Endocrine Revs 1993; 14: 459-479.

19. Yang-Yen H-F, Chambard J-C, Sun Y-L, et al. Transcriptional interference between c-Jun and the glucocorticoid receptor: mutual inhibition of DNA-binding due to direct protein-protein interaction. Cell 1990; 62: 1205-1215.

20. Adcock IM, Lane SJ, Brown CR, Peters MJ, Lee TH, Barnes PJ. Differences in binding of glucocorticoid receptor to DNA in steroid-resistant asthma. J Immunol 1995; 154: 3500-3505.

21. Klemm DJ, Surs W, Spahn JD, Szefler SJ, Leung DYM. Alterations in glucocorticoid receptor-binding to DNA contributes to steroid-resistant asthma. J Allergy Clin Immunol 1996; 97: 716A.

22. Bamberger CM, Bamberger A-M, de Castro M, Chrousos GP. Glucocorticoid receptor- $\beta$, a potential endogenous inhibitor of glucocorticoid action in humans. J Clin Invest 1995; 95: 2435-2441.

23. Lane SJ, Atkinson BA, Swaminathan R, Lee TH. Hypothalamic-pituitary-adrenal axis in corticosteroid-resistant bronchial asthma. Am J Respir Crit Care Med 1996; 153 : 557-560.

24. Nimmagadda SR, Leung DYM, Spahn JD, Surs W, Szefler SJ. Mechanisms underlying allergen-induced glucocorticoid receptor-binding abnormalities. J Allergy Clin Immunol 1995; 95: 64A. 
25. Leung DYM, Martin RJ, Szefler SJ, et al. Dysregulation of interleukin-4, interleukin-5, and interferon-gamma gene expression in steroid-resistant asthma. J Exp Med 1995; 181: 33-40.

26. D'Alonzo GE, Smolensky MH, Feldman S, et al. Twenty four hour lung function in adult patients with asthma: chronoptimized theophylline therapy once daily dosing in the evening versus conventional twice daily dosing. Am Rev Respir Dis 1990; 142: 84-90.

27. Martin RJ, Cicutto LC, Ballard RD, Goldenheim PD, Cherniack RM. Circadian variations in theophylline concentrations and the treatment of nocturnal asthma. Am Rev Respir Dis 1989; 139: 475-478.

28. Kossoy AF, Hill MR, Lin FL, Szefler SJ. Are theophylline "levels" a reliable indicator of compliance? $J$ Allergy Clin Immunol 1989; 84: 60-65.

29. Reinberg A, Halberg F, Falliers CJ. Circadian timing of methylprednisolone effects in asthmatic boys. Chronobiologia 1974; 1: 333-347.

30. Kharitonov SA, Yates DH, Chung YK, Barnes PJ. Changes in the dose of inhaled steroid affect exhaled nitric oxide levels in asthmatic patients. Eur Respir J 1996; 9: 196-201.

31. Corrigan CJ, Brown PH, Barnes NC, et al. Glucocorticoid resistance in chronic asthma: peripheral blood $\mathrm{T}$ lymphocyte activation and comparison of the T-lymphocyte inhibitory effects of glucocorticoids and cyclosporin A. Am Rev Respir Dis 1991; 144: 1026-1032.

32. Pedersen S, Hansen OR. Budesonide treatment of moderate and severe asthma in children: a dose-response study. J Allergy Clin Immunol 1995; 95: 29-33.

33. Johnson M. Pharmacodynamics and pharmacokinetics of inhaled glucocorticoids. J Allergy Clin Immunol 1996; 97: 169-176.

34. Noonan M, Chervinsky P, Busse WW, et al. Fluticasone propionate reduces oral prednisone use while it improves asthma control and quality of life. Am J Respir Crit Care Med 1995; 152: 1467-1473.

35. McCubbin MM, Milavetz G, Grandgeorge S, et al. A bioassay for topical and systemic effect of three inhaled corticosteroids. Clin Pharmacol Ther 1995; 57: 455-460.

36. Robinson DS, Geddes DM. Inhaled corticosteroids: benefits and risks. J Asthma 1996; 3: 5-16.

37. Toogood JH, Baskerville J, Jenning B, Lefcoe NM, Johansson S-A. Bioequivalent doses of budesonide and prednisone in moderate and severe asthma. $J$ Allergy Clin Immunol 1989; 84: 688-700.

38. Agertoft L, Pedersen S. Importance of the inhalation device on the effect of budesonide. Arch Dis Child 1993; 69: 130-133.

39. Thorsson L, Edsbacker S, Conradson T-B. Lung deposition of budesonide from Turbuhaler is twice that from a pressurized metered dose inhaler. Eur Respir J 1994; 7: $1839-1844$.

40. Szefler SJ. Anti-inflammatory drugs in the treatment of allergic disease. Med Clin North Am 1992; 76: 953-975.

41. Jarjour N, McGill K, Busse WW, Gelfand E. Alternative anti-inflammatory and immunomodulatory therapy. In: Szefler SJ, Leung DYM, eds. Severe Asthma: Pathogenesis and Clinical Management. Lung Biology in Health and Disease. Vol. 86. New York, Marcel Dekker, 1996; pp. 333-369.

42. Finnerty JP, Wood-Baker R, Thomson H, Holgate ST. Role of leukotrienes in exercise-induced asthma: inhibitory effect of ICI 204,219, a potent leukotriene $\mathrm{D}_{4}$ receptor antagonist. Am Rev Respir Dis 1992; 145: 46-49.

43. Taylor IK, O'Shaughnessy KM, Fuller RW, Dollery CT. Effect of cysteinyl-leukotriene receptor antagonist, ICI 204,219 on allergen-induced bronchoconstriction and airway hyperreactivity in atopic subjects. Lancet 1991; 337: 690-694.

44. Israel E, Rubin P, Kemp JP, Grossman J, et al. The effect of inhibition of 5-lipoxygenase by Zilueton in mild-to-moderate-asthma. Ann Intern Med 1993; 119: 1059-1066.

45. Hegele RG, Hogg JC. The pathology of asthma: an inflammatory disorder. In: Szefler SJ, Leung DYM, eds. Severe Asthma: Pathogenesis and Clinical Management. Lung Biology in Health and Disease. Vol. 86. New York, Marcel Dekker, 1996; pp. 61-76. 